\section{Géneros}

Multidisciplinary Journal of Gender: Studies

\section{Hipatia Press \\ www.hipatiapress.com}

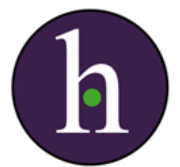

Instructions for authors, subscriptions and further details:

$\underline{\text { http://generos.hipatiapress.com }}$

\title{
Analizando la Masculinidad en la Ley General de la Vivienda Cubana para la Formación Profesional de Juristas
}

Jorge Luis Silva-González ${ }^{1}$

Alie Pérez-Véliz ${ }^{1}$

Yudmila Delgado-Rodríguez ${ }^{1}$

1) Universidad "Hermanos Saíz Montes de Oca”, Cuba.

Date of publication: February $25^{\text {th }}, 2018$

Edition period: February - June 2018

To cite this article: Silva-González, J.L., Pérez-Véliz, A. \& Delgado-

Rodríguez, Y. (2017). Analizando la Masculinidad en la Ley General de la Vivienda Cubana para la Formación Profesional de Juristas.

Multidisciplinary Journal of Gender Studies, 7(1), 1486-1505. doi:

10.17583/generos.2018.3132

To link this article: http://dx.doi.org/10.17583/generos.2018.3132

\section{PLEASE SCROLL DOWN FOR ARTICLE}

The terms and conditions of use are related to the Open Journal System and to Creative Commons Attribution License (CC-BY). 


\section{Analyzing masculinity in the General Law of Cuban Housing for professional training of jurists}

Jorge Luis Silva-González

Alie Pérez-Véliz

Yudmila Delgado-Rodríguez

Universidad "Hermanos Saiz Montes de Oca"

\section{Abstract}

The study emphasizes the need to train the student of Law in Cuba in the subject Law on Property, so that once graduated in the University in legal practice, interpret and apply the Law from the perspective of equity, in relative cases to the legal limits to the right of the owner to declare the cessation of cohabitation against those who inhabit his dwelling. Its regulation in the General Housing Law offers a discriminatory treatment for the male sex in relation to the female sex, when this is not the owner of the house, being in a position definable by the court and before the case of injustice or act inhumane, since the normative provision does not protect it.

Keywords: training, gender, masculinity, law 


\section{Analizando la Masculinidad en la Ley General de la Vivienda Cubana para la Formación Profesional de Juristas}

Jorge Luis Silva-González

Alie Pérez-Véliz

Yudmila Delgado-Rodríguez

Universidad "Hermanos Saíz

Montes de Oca”

\section{Resumen}

El estudio enfatiza en la necesidad de formar al estudiante de Derecho en Cuba en la asignatura Derecho sobre Bienes, para que una vez egresado de la Universidad en la práctica jurídica, interprete y aplique el Derecho desde la perspectiva de la equidad, en los casos relativos a los límites legales al derecho del propietario a declarar el cese de convivencia contra quienes habitan su morada. Su regulación en la Ley General de la Vivienda ofrece un tratamiento discriminatorio para el sexo masculino en relación con el sexo femenino, cuando este no es el propietario de la vivienda, quedando en una posición definible por el tribunal y ante el supuesto de injusticia o acto inhumano, ya que la disposición normativa no lo protege.

Palabras clave: formación, género, masculinidad, derecho

2018 Hipatia Press

ISSN: 2014-3613

doi: $10.17583 /$ generos.2018.3132 


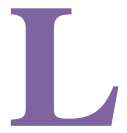

a formación de profesionales competentes se ha convertido en un reclamo de la sociedad contemporánea y en tal sentido, las investigaciones educativas poseen una relevancia capital si de perfeccionamiento de la Educación Superior se trata. La creación de investigaciones interdisciplinarias que tributen al desarrollo óptimo del proceso de enseñanza-aprendizaje, amerita un espacio significativo en los estudios científicos (Silva \& Pérez, 2017).

En Cuba, dicha formación, teniendo en cuenta el enfoque de género desde la perspectiva de la equidad en la legislación imperante, es centro de atención para quienes investigan en la carrera de Derecho -relacionando las Ciencias Pedagógicas con las Ciencias Jurídicas-, con el propósito de graduar profesionales que cumplan con el encargo de alcanzar mayor justicia social, atendiendo a las brechas de desigualdad que presentan algunas disposiciones normativas del ordenamiento jurídico que, como se evidencia en el presente artículo, necesita ser perfeccionado a la luz de los avances actuales en materia de género y el contexto existente.

Se considera, que cada jurista necesita ser dotado -desde el propio proceso de enseñanza-aprendizaje llevado a cabo por la Universidad como centro rector de la Educación Superior-, de las herramientas indispensables para interpretar y aplicar la ley en la práctica jurídica, de forma que no se privilegie a un sexo por encima del otro, a partir de la propia inequidad existente aún entre hombres y mujeres en la sociedad.

En tal sentido, es preciso plantear que el ordenamiento jurídico cubano, está elaborado sobre la base del Principio de Igualdad establecido en la vigente Constitución de la República de 1976, al hacer alusión en los artículos 41 y 43 respectivamente, a que todos los ciudadanos gozan de iguales derechos y están sujetos a iguales deberes; asimismo que, la mujer y el hombre gozan de iguales derechos en lo económico, político, cultural, social y familiar.

Sin embargo, a pesar de la importancia que reviste este principio del Derecho, transversal a las disposiciones normativas del ordenamiento jurídico, una revisión exploratoria a la Ley No. 65, Ley General de la Vivienda, de 23 de diciembre de 1988, permitió identificar que presenta un enfoque de género que no se ajusta al mismo y por tanto, no es equitativo para ambos sexos, en lo relativo a los límites legales al derecho del 
propietario, según lo previsto de la Sección Primera "Derechos y obligaciones de los Propietarios".

Teniendo en cuenta lo anteriormente expuesto, el presente estudio tiene como objetivo: contribuir a la formación del profesional del Derecho en Cuba en la asignatura Derecho sobre Bienes, sobre la base del análisis del enfoque de género desde la perspectiva de la equidad, en la actual Ley General de la Vivienda cubana, en lo relativo a los límites legales al derecho del propietario, según lo previsto de la Sección Primera "Derechos y obligaciones de los Propietarios”, específicamente en los artículos 64 y 65, lo que posibilitará la superación del enfoque sexista, tradicionalista y biologicista de dicha norma en la interpretación y aplicación del Derecho en la práctica jurídica sin necesidad de transformar la ley.

De esta manera, el profesorado que imparte la asignatura Derecho sobre Bienes y el estudiantado que la recibe, podrán profundizar en el tema y alcanzar mayor conciencia jurídica acerca de la importancia de la equidad, en la interpretación y aplicación de la ley, en los casos donde se ejercite el derecho del propietario a declarar el cese de convivencia contra quienes habitan su morada por la vía judicial y no precisamente como establece la normativa cubana de manera tradicionalista, sexista y biologicista, como se apreciará más adelante en el análisis desarrollado.

Para ello, se particulariza especialmente en la universidad como centro educativo, con énfasis en la triada formación del profesional-género-equidad y se hace referencia al proceso de formación del profesional en Cuba, a la teoría sobre género y equidad, con énfasis en el enfoque de género y, atendiendo a que el estudio que se presenta es de tipo teórico, con un alcance descriptivo-correlacional, que busca no solo exponer las características, propiedades y manifestaciones del género, recolectando, ordenando y jerarquizando información, estableciendo relaciones, sino que en el análisis de la interactuación que tienen en el objeto de estudio determinadas variables o categorías, logra aclarar la incidencia de estas en su manifestación y regularidades de comportamiento.

En la investigación se requirió el empleo de métodos que posibilitaron los análisis del contenido, tales como el análisis jurídico doctrinal y el exegético analítico que fue esencial para el análisis de la disposición normativa. 
Por lo antes expuesto, el estudio enfatiza en la necesidad de superar el actual enfoque de género, en la rama del Derecho sobre Bienes en Cuba, desde una perspectiva diferente, que brinde como corolario equidad legislativa para el sexo masculino en relación con el sexo femenino desde la actuación de juristas formados para ello.

\section{La formación del profesional y sus particularidades en la asignatura Derecho sobre Bienes}

La formación de profesionales del Derecho en Cuba está determinada por el papel que juega la Universidad en el proceso de preparación del estudiantado en cada esfera de la vida social, y se puede catalogar como integral.

"El vocablo proceso proviene del latín procesus, que significa paso, avance" (Páez, 2014, p. 18). Según el Diccionario de la Lengua Española (2014), es la acción de ir hacia delante, el conjunto de las fases sucesivas de un fenómeno natural o de una operación artificial. Para Álvarez (1999) es una sucesión de estados de un objeto determinado, tiene que ver con la importante función de preparar al hombre en todos los aspectos de su personalidad.

En la Educación Superior cubana, el término formación se emplea para definir el proceso sustantivo desarrollado en las universidades con el objetivo de preparar integralmente a cada estudiante en una determinada carrera universitaria y abarca, tanto los estudios de pregrado como los de postgrado (Horruitinier, 2007). "Es el proceso totalizador cuyo objetivo es preparar al hombre como ser social" (Álvarez de zayas, 1999, p. 9).

A partir de los criterios anteriores, el proceso de formación de juristas en materia de Derecho sobre Bienes y posteriormente su superación, una vez egresados de la institución docente, es un tema significativo que adquiere dimensiones ilimitadas en los marcos intra y extra universitarios.

Siguiendo la lógica de los criterios abordados y atendiendo al objetivo del presente estudio, la carrera de Derecho se concibe, desde la concepción del plan de estudios vigente, Plan D perfeccionado, para formar profesionales competentes, capaces de interpretar, aplicar, crear y/o modificar, no solo las leyes, sino la realidad social en que se desenvuelven. Es por ello que resulta estratégico desde el punto de vista político la 


\section{Silva, Pérez \& Delgado - Analizando la Masculinidad}

incorporación del enfoque de género de forma consciente en la preparación de quienes egresarán de la carrera.

En tal sentido, el profesorado juega un papel decisivo, en tanto están encargados de llevar a las aulas los análisis pertinentes de cada una de las materias o asignaturas. Para ello, se auxiliarán de la Pedagogía y la Didáctica. A los efectos de la investigación, se centrará la atención en la asignatura Derecho sobre Bienes, que es la que tiene como objeto propio de estudio las relaciones jurídicas en el ámbito referente a los bienes inmobiliarios y donde se encuentra la vivienda.

El estudio del régimen jurídico de las viviendas de propiedad personal y las relaciones jurídicas sobre bienes inmuebles, que se manifiestan en el tráfico jurídico, tomando como base los derechos, facultades, acciones y deberes reales, así como los medios jurídicos idóneos para su ejercicio, en el proceso de formación de profesionales del Derecho en Cuba, corresponde a la asignatura de Derecho sobre Bienes.

Esta asignatura forma parte de la disciplina de Derecho Civil y de Familia, y tiene como objetivo general: fundamentar las relaciones jurídicas sobre bienes muebles e inmuebles, que se manifiestan en el tráfico jurídico utilizando para ello los derechos, facultades, deberes y acciones, todos de carácter real, así como los medios jurídicos idóneos para su ejercicio, mediante la lectura de textos, análisis y discusión de documentos y normas jurídicas, revisión y estudio de casos problemáticos, además de debates en grupo, haciéndolo con un alto sentido de la responsabilidad, el humanismo, la legalidad, el respeto a los derechos de las personas y la defensa de la propiedad socialista.

Se ubica en el segundo semestre del segundo año de la carrera y cuenta con un fondo de tiempo de 64 horas presenciales. Para el desarrollo de la misma, el contenido se divide en dos unidades didácticas. La primera responde a los fundamentos teórico-jurídicos sobre los derechos reales, donde cada estudiante debe estar en condiciones de explicar los derechos, facultades, deberes y acciones reales, tomando como base los principios, categorías e instituciones que giran en torno a ellos, a través de la lectura de textos, análisis y discusión de documentos y normas jurídicas, revisión y estudio de casos problemáticos, además de debates grupales; haciéndolo con un alto sentido de la responsabilidad, el respeto a los derechos de las personas, la legalidad, el humanismo y la defensa de la propiedad socialista, 
y la segunda unidad didáctica, al Derecho inmobiliario cubano, que se toma como base para el estudio y es a la cual trascenderá el resultado del mismo.

En esta última, según el programa de la asignatura, el estudiantado debe valorar desde el punto de vista jurídico el Derecho inmobiliario cubano, a través de la lectura de textos, el análisis y la discusión de documentos y normas jurídicas, la revisión y el estudio de casos problemáticos, además de debates grupales; haciéndolo con un alto sentido de la responsabilidad, el respeto a los derechos de las personas, la legalidad, el humanismo y la defensa de la propiedad socialista.

Según lo planteado, pudiera parecer que la organización del contenido carece de la inclusión del análisis del enfoque de género y sin embargo no es así, es en cada una de las formas de clases donde el profesorado define, a través del sistema de conocimientos que se va a abordar, el tratamiento a seguir en cuanto al enfoque de género (tratado en el próximo epígrafe) en el desarrollo de las relaciones familiares asociadas a la vivienda como bien inmueble, las cuales están marcadas por un desbalance en la jerarquía mujer vs hombre, según las etapas del desarrollo de las fuerzas productivas y donde el hombre como jefe del hogar tiene también el poder sobre la propiedad que comparte con su mujer e hijos/as.

La Ley No. 65, Ley General de la Vivienda, de 23 de diciembre de 1988, modificada por los decretos-leyes Nos. 185 de 28 de mayo de 1998; 233 de 2 de julio de 2003; 288 de 28 de octubre de 2011; 322 de 31 de julio de 2014 y 342 del 14 de diciembre de 2016, en la Sección Primera plantea los derechos y obligaciones de los propietarios de una vivienda y el resto de la familia o personas que la habitan.

Atendiendo a los criterios anteriores, se considera que antes de exponer el análisis del enfoque de género en la mencionada Ley 65 , se debe hacer referencia a las categorías de género y equidad, sobre cuya esencia o contenido se fundamentó el estudio.

\section{Reflexiones sobre género y equidad de género}

El género como concepto, para la investigadora Norma Vasallo (2004) es de temprana incorporación al análisis y debate científico. La categoría empezó a ser utilizada en las Ciencias Sociales en la década de los setenta en los países del primer mundo (Estados Unidos y Europa), extendiéndose más 


\section{Silva, Pérez \& Delgado - Analizando la Masculinidad}

tarde a América Latina y el Caribe, África y Asia; pero se reconoce su origen en la obra del sicólogo de Nueva Zelandia, Jhon Money, quien en 1951, usa el concepto gender por primera vez para referirse a un componente cultural, fundamentalmente a la influencia educativa, en la formación de la identidad sexual (Pautassi, 2012).

En el idioma español, según Lamas (2014), la definición clásica de diccionario, se refiere al género como la clase, especie o tipo a la que pertenecen las personas o las cosas. Plantea que el Diccionario del uso del español, de María Moliner consigna cinco acepciones de género y apenas la última es la relativa al género gramatical, o sea, a la definición gramatical por la cual los sustantivos, adjetivos, artículos o pronombres pueden ser femeninos, masculinos o -sólo los artículos y pronombres- neutros.

Refiere la mencionada investigadora, que según María Moliner, tal división responde a la naturaleza de las cosas sólo cuando esas palabras se aplican a animales, pero a los demás se les asigna género masculino o femenino de manera arbitraria. Infiere que:

En castellano la connotación de género como cuestión relativa a la construcción de lo masculino y lo femenino sólo se comprende en función del género gramatical, y sólo las personas que ya están en antecedentes del debate teórico al respecto lo comprenden como la simbolización o construcción cultural que alude a la relación entre los sexos (Lamas, 2014, p. 2).

Para Lamas, la primera disciplina que empleó la categoría género, para establecer una diferencia con el sexo fue la Psicología, en su vertiente médica, por Robert Stoller (1968) quien estudió los trastornos de la identidad sexual, examinando casos en los que la asignación de sexo falló, ya que las características externas de los genitales se prestaban a confusión. Según Izquierdo (1998), tanto para Stoller como para Money, se evidenciaban dos cuestiones fundamentales:

- Algunos individuos no podían ser clasificados en machos o hembras desde el punto de vista del dimorfismo sexual porque poseían los caracteres sexuales secundarios poco marcados.

- Otras personas que morfológicamente se encontraban bien definidas sexualmente, declaraban sentirse en un cuerpo equivocado. 
Desde esta perspectiva psicológica, para Lamas, el género es una categoría en la que se articulan tres instancias básicas:

- La asignación (rotulación, atribución) de género, que se realiza en el momento en que nace el bebé, a partir de la apariencia externa de sus genitales.

- La identidad de género, que se establece más o menos a la misma edad en que el infante adquiere el lenguaje (entre los dos y tres años) y es anterior a su conocimiento de la diferencia anatómica entre los sexos. Desde dicha identidad, el niño estructura su experiencia vital; el género al que pertenece lo hace identificarse en todas sus manifestaciones: sentimientos o actitudes de niño o de niña, comportamientos, juegos, etcétera.

- El papel de género, que se forma con el conjunto de normas y prescripciones que dictan la sociedad y la cultura sobre el comportamiento femenino o masculino. Se puede sostener una división básica que corresponde a la división sexual del trabajo más primitiva: las mujeres paren a los hijos, y por lo tanto, los cuidan: ergo, lo femenino es lo maternal, lo doméstico, contrapuesto con lo masculino como lo público.

Lo que el concepto de género ayuda a comprender es que muchas de las cuestiones que se piensan que son atributos naturales de los hombres o de las mujeres, en realidad son características construidas socialmente, que no tienen relación con la biología. El trato diferencial que reciben niños y niñas, sólo por pertenecer a un sexo, va generando una serie de características y conductas diferenciadas (Lamas, 2014, p.4).

Dicha definición se relaciona con el criterio de Lagarde (1994), que concibe también al género como el conjunto de características -asignadas a cada sexo diferencialmente-, y de normas sociales, económicas, políticas, culturales, psicológicas, jurídicas.

"Tanto Scout (1990) y Lagarde (1994), como Miranda y Peña (2001), Proveyer (2005) y Caram (2016), coinciden con que el género es una construcción social que se basa en diferencias biológicas" (Silva, Pérez \& Páez, 2017).

De acuerdo con la consideración anterior que se vislumbraba desde la década de los años 90, la V Conferencia Internacional de la Mujer celebrada en Beijing, China, en 1995, acordó que la palabra género se diferencia de 
sexo para expresar que el rol y la condición de hombres y mujeres responden a una construcción social y están sujetos a cambios.

Para Valdebenito (2002), las diferencias entre los hombres y las mujeres son de naturaleza biológica y de carácter social:

El término sexo se refiere a unas diferencias biológicamente determinadas con carácter universal entre los hombres y las mujeres. El término género se refiere a unas diferencias sociales y relacionadas entre los hombres y las mujeres aprendidas, cambiantes con el tiempo y que presentan una gran variabilidad entre las diversas culturas y aun dentro de una misma cultura. Estas diferencias y relaciones son una construcción social, y han sido aprendidas a través del proceso de socialización. Son específicas de un contexto y pueden ser modificadas (p.44).

Por su parte, Vasallo en el 2004, plantea que la formación y desarrollo del género dependen de procesos culturales, del entorno de desarrollo de las personas desde el mismo momento de su nacimiento. Refería Caram en 1996, en que es un patrón cultural y diez años después -en el 2016-, plantea que en cada sociedad tiene su propuesta de modelos para los sexos y que pueden variar a través del tiempo; encontrando su origen en las definiciones sociales y culturales que rigen la conducta de las personas.

Atendiendo a las definiciones anteriores, resulta interesante y a la vez significativo, como desde la década de los años 90 existe consenso en la doctrina, en torno a una definición de género similar y se asume en la investigación al género como un proceso de construcción social y/o cultural que asigna a cada persona según su sexo -desde el momento de su nacimiento-, características, roles, valores y normas de todo tipo, ya sean sociales, políticas, económicas, jurídicas, culturales y psicológicas.

Asimismo, se considera que sexo y género no significan lo mismo, en tanto el primero se refiere a las condiciones anátomo-fisiológicas (Miranda \& Peña, 2001; Proveyer, 2005) del ser humano.

En cuanto al enfoque de género, este se concibe según Ahr (2007), como los diferentes papeles que la sociedad asigna a mujeres y hombres y que se reflejan, en la división y carga de trabajo, en el diferente acceso a los recursos y el desigual control sobre ellos, así como en las distintas posibilidades que tienen las personas de ejercer influencia política y social. 
Se considera, que para llevar a cabo el análisis del enfoque de género, la finalidad debe estar siempre dirigida a disminuir o erradicar las brechas de desigualdad, o sea, lograr justicia y equidad, entre hombres y mujeres.

Según Valdebenito (2002) la equidad supone un disfrute de los bienes sociales, recursos, las mismas oportunidades en la toma de decisiones, y trabajar juntos ambos sexos para lograrlo.

Dicha equidad como se mencionó, no es sinónimo de igualdad con respecto al género. Para la autora anterior, las responsabilidades y las oportunidades de las mujeres y de los hombres no dependan de que hayan nacido mujeres u hombres; es un problema de derechos humanos y una condición previa para la consecución de un desarrollo sostenible centrado en las personas. Según consta en Relaciones de género con equidad: Guía Conceptual y Metodológica:

"Equidad: No es igualdad. La equidad es la distribución justa de acuerdo a los intereses y necesidades de hombres y mujeres. La equidad es una fórmula donde todas las partes salgan ganando aunque sus intereses y necesidades sean diferentes. Es satisfacer individualidades por un bienestar colectivo (Miranda \& Peña, 2001, p. 77).

No alejada del criterio anterior y tempranamente, la primera Conferencia Mundial sobre la Mujer, en México (1975) que culminó con la propuesta de un Plan de Acción aprobado por la Asamblea General de las Naciones Unidas, acordó que la igualdad es al mismo tiempo un objetivo y un medio por el cual los individuos se benefician del mismo trato en el marco de la ley y de las mismas oportunidades para gozar sus derechos y desarrollar sus talentos y habilidades de manera que puedan participar en el desarrollo político, económico, social y cultural, tanto como beneficiarios que como agentes activos.

En tanto Valdebenito (2002) coincide con la definición anterior al plantear, la "igualdad de derechos significa igualdad formal ante la ley. Equiparación de hombres y mujeres mediante medidas legislativas" (p. 37). Dicha igualdad debe estar establecida o amparada "en principio" por la Constitución de cada país, y como ya se planteó en la introducción del estudio, Cuba la regula en el Capítulo VI del artículo 41 al 44. 
En cuanto a la igualdad de género "se entiende como una relación de equivalencia en el sentido de que las personas tienen el mismo valor, independientemente de su sexo, y por ello son iguales" (Valdebenito, 2002, p. 37).

Coincide Camargo (1999), que desde el punto de vista del Derecho, "establece una teoría social que trata de explicar (...) comportamientos sociales de hombres y mujeres en sociedad, (...) destacando la existencia real del género femenino y masculino, sin dominio de uno sobre el otro, sin jerarquías y sin desigualdades" (p. 29).

Para la abogada Staff (1999), significa la promoción de la igualdad y de las nuevas identidades de género, reduciendo o eliminando las causas y efectos de la discriminación en el ámbito jurídico.

Se está de acuerdo con Miranda y Peña (2001) y Valdebenito (2002), en que la equidad de género no supone que hombres y mujeres sean iguales, pero sí que tengan acceso y disfruten de los bienes sociales, oportunidades y recursos sin distinción alguna, sin que un sexo prevalezca por encima del otro.

El énfasis en la igualdad y en el empoderamiento de género, no presupone un modelo definitivo de igualdad para todas las sociedades, pero refleja la preocupación de que ambos sexos, aunque no sean iguales por razones biológicas, sí tengan las mismas facilidades para tomar decisiones y trabajen juntos para lograrlo en virtud del principio de equidad.

A tenor de la investigación, se entenderá por enfoque de género en el Derecho sobre Bienes inmobiliarios, desde la perspectiva de la equidad, la posición y el tratamiento que ofrece para hombres y mujeres el conjunto de normas que dan orden estructural y funcional a esta rama del Derecho, sobre la base de un equilibrio en el que, ninguno de ambos sexos se beneficie de manera injusta por encima del otro. Dicha definición se tiene en cuenta en el análisis que se ofrece a continuación.

\section{Análisis del Enfoque de Género en la Ley General de la Vivienda Cubana}

La Ley No. 65 de 1988, Ley General de la Vivienda cubana en lo relativo a los límites legales al derecho del propietario, según lo previsto de la Sección Primera "Derechos y obligaciones de los Propietarios" regula que: 
Artículo 64: Los propietarios de las viviendas determinarán libremente qué personas convivirán con ellos y estarán facultados para dar por terminada la convivencia de cualquiera persona, para lo que no requerirán declaración administrativa ni judicial (...).

Artículo 65: La facultad que concede al propietario el artículo precedente no podrá ejercerse contra:

Ascendientes y descendientes del propietario:

$\checkmark$ Madre con uno o más hijos habidos en el matrimonio, formalizado o no, con el propietario siempre que ella tenga la guarda y cuidado de los hijos y no tuviere otro lugar de residencia;

$\checkmark$ Madre con uno o más hijos menores que lleven tres o más años ocupando la vivienda y no tuvieren otro lugar de residencia;

$\checkmark$ Ancianos que lleven tres o más años ocupando la vivienda y no tuvieren otro lugar de residencia;

$\checkmark$ Cualquier otro caso que a juicio de la autoridad competente constituya una manifiesta injusticia o acto inhumano.

De acuerdo a lo establecido por las disposiciones precedentes, el propietario puede ejercer el cese de convivencia contra todos los que habitan su morada, excepto contra la madre de sus hijos, si no tuviere otro lugar de residencia; madre con hijos menores que lleven tres años o más en su vivienda, y que cumplan la condición anterior de no poseer otra residencia; además de ascendientes y descendientes del propietario, o casos de clara injusticia valorados por la autoridad competente.

Se considera que dicha disposición normativa responde a un contexto histórico-social en Cuba, que mejoró significativamente la posición de la mujer, atendiendo a que la misma fue aprobada y puesta en vigor a finales de la década de los 80, un período en el que se promulgó el Código de Trabajo de 1984 y el Reglamento para la Política del Empleo de 1987, como parte de las acciones revolucionarias para ofrecer garantías y oportunidades a las mujeres, completándose una legislación que ofrecía derechos en los planos 
personal, patrimonial, laboral, sexual, reproductivos y filiales, sobre todo en lo referente a la protección de la madre trabajadora (Silva \& Pérez, 2017).

Es justa la regulación del legislador cubano para la figura materna y sus hijos, partiendo de que responde a la necesidad de prestar especial protección no solo a esta (quien asumía el rol socialmente atribuido de cuidar a sus hijos) sino a los propios hijos menores habidos en el matrimonio o no, a fin de garantizarles un lugar seguro para sus vidas donde pudieran habitar.

Como se evidencia en el artículo 65, la norma protege a la mujer madre de menores y lo hace sin necesidad de prueba y por formulación taxativa, prejuzgando en su favor cualquier fallo administrativo por decisión del legislador. Sin embargo, al llevar a cabo un análisis del enfoque de género en la disposición, en el caso de que la situación fuera contraria y la propietaria fuera la mujer -con sus respectivos derechos declarados en el cuerpo de la norma-, el hombre padre de hijos menores casado con esta, estaría en una condición solo definible por decisión de la autoridad competente, y ante el supuesto de injusticia o acto inhumano.

Con dicha regulación no se establece garantía alguna para el sexo masculino, aun cuando la propia disposición en su último acápite ampara cualquier otro caso que a juicio de la autoridad competente constituya una manifiesta injusticia o acto inhumano, condición que le ofrece al Órgano de la Dirección Municipal de la Vivienda -competente en estos asuntos-, la facultad discrecional de decidir a favor del hombre, aun cuando no figura entre los especialmente protegidos por la norma.

También se considera que la misma es tradicionalista, sexista, al estar basada en el prejuicio socialmente reconocido, que supone el ser hombre (fuerte, independiente económicamente, educador a distancia de sus hijos) ante el ser mujer (frágil, dependiente económicamente, responsable del cuidado de sus hijos), quien además hallará siempre una solución en tal circunstancia como "macho, varón, masculino" cuando no tuviere otro lugar de residencia.

Es esta una regulación que además valora a todos los hombres por igual atendiendo a su capacidad física y psíquica para afrontar tal supuesto de hecho, cuando la realidad manifiesta que estamos en presencia de una sociedad que tiende a la modernización de estos patrones y es muchas veces el padre el que asume la guarda y cuidado de los hijos por diversas causas, 
entre las que se encuentran: la enfermedad de la madre, su fallecimiento y el cumplimiento de colaboración extranjera.

También es preciso señalar, cómo la norma se ha mantenido durante décadas, cuando la vivienda aún no es un problema resuelto en Cuba y muchas familias se componen no solo por padre, madre e hijos (núcleo básico), sino que a ellos se suman abuelos, hermanos y nietos.

Se sugiere en consonancia con los análisis anteriores, que la disposición normativa del artículo 65 (excluyente para el padre de hijos no propietario de vivienda y sin otro lugar de residencia una vez disuelto el vínculo matrimonial), sea modificada a través de un Decreto - Ley, y sea interpretada o valorada en la práctica jurídica actual -en los procesos llevados a cabo por la vía judicial-, de forma que se proteja también al padre, haciendo un uso extensivo de la norma, como se plantea a continuación:

Artículo 65: La facultad que concede al propietario (a)el artículo precedente (cese de convivencia, artículo 64) no podrá ejercerse contra:

Ascendientes y descendientes del propietario (a):

$\checkmark$ Madre o padre con uno o más hijos (as) habidos (as) en el matrimonio, formalizado o no, con el propietario (a) siempre que ella o él tenga la guarda y cuidado de los hijos y no tuviere otro lugar de residencia;

$\checkmark$ Madre o padre con uno (a) o más hijos (as) menores que lleven tres o más años ocupando la vivienda y no tuvieren otro lugar de residencia.

El análisis desarrollado debe tenerse en consideración en la impartición de la asignatura Derecho sobre Bienes, de modo que se pueda suplir desde la docencia y para la práctica jurídica dicha brecha de desigualdad que nada tiene que ver con el Principio de Igualdad reconocido en la vigente Constitución de la República que data de 1976, y que pone en una posición desventajosa al hombre padre cuando disuelto el vínculo matrimonial no tenga este otro lugar de residencia.

El mismo puede ser incorporado atendiendo a las particularidades del programa de estudio de la asignatura antes mencionada, en el contenido de la Unidad Didáctica II: El Derecho Inmobiliario cubano, específicamente en la 


\section{Silva, Pérez \& Delgado - Analizando la Masculinidad}

Conferencia 4: Régimen Jurídico de las viviendas de propiedad personal, que dentro del sumario -como se aprecia a continuación-, se imparte en el tópico d) en la carrera de Derecho de la Universidad Hermanos Saíz Montes de Oca de Pinar del Río, donde se llevó a cabo el estudio.

Sumario:

a) Principios legales sobre los bienes inmuebles urbanos de propiedad personal

b) Adquisiciones y transmisiones de viviendas de propiedad personal

c) Adquisiciones resultantes de la Ley. La ocupación

d) Derecho del propietario a declarar la convivencia y su cese

e) Ocupación Ilegal

Dicha inclusión es significativa porque una vez graduado el profesional del Derecho en la práctica jurídica -sin necesidad de transformar la ley o hacer un cambio legislativo-, puede en la interpretación y aplicación de la disposición normativa, haciendo uso de la aplicación evolutiva del Derecho, incorporar el adelanto científico obtenido en la actualidad a normas como estas que se corresponden y aprobaron en un contexto histórico de escaso progreso teórico para la categoría género, específicamente incorporada en la década de los años setenta en las Ciencias Sociales.

\section{Conclusiones}

La enseñanza del Derecho sobre Bienes desde la perspectiva de la equidad, reviste especial importancia para la carrera de Derecho en Cuba, en función de la formación de profesionales que sean capaces de lograr en la práctica jurídica -una vez egresados de la Universidad- mayor justicia social en cuanto a las relaciones jurídicas y el ejercicio de derechos y obligaciones que se ejercitan entre hombres y mujeres, en lo relativo a las disposiciones -y/o conflictos que se suscitan- en materia de la vivienda.

Asumido en la investigación al enfoque de género en el Derecho sobre Bienes inmobiliarios, desde la perspectiva de la equidad, como la posición y el tratamiento que ofrece para hombres y mujeres el conjunto de normas que dan orden estructural y funcional a esta rama del Derecho, sobre la base de un equilibrio en el que, ninguno de ambos sexos se beneficie de manera injusta por encima del otro; el análisis desarrollado arroja que actualmente las disposiciones normativas al respecto, en la Ley General de la Vivienda, 
en lo relativo a los límites legales al derecho del propietario, según lo previsto de la Sección Primera "Derechos y obligaciones de los Propietarios" son discriminatorias, ya que la norma ante la voluntad expresa del cese de convivencia del propietario para quienes habiten su morada, protege a la mujer madre de hijos menores cuando no tuvieren otro lugar de residencia, contrario sensu del hombre, que en la misma condición (padre de hijos menores sin otro lugar de residencia) quedaría en una posición definible por el tribunal y ante el supuesto de injusticia o acto inhumano, ya que la disposición normativa no lo contempla o protege. Es esta una realidad que se convierte en un reto para quienes operan el Derecho en Cuba, dígase jueces, fiscales y abogados relacionados con esta rama, si se pretende como premisa fundamental contribuir a la justicia social y a la equidad de género desde la interpretación y aplicación de la ley.

A partir de lo planteado en el estudio, se evidencia que la Universidad cubana está expuesta a desafíos tanto curricular como extracurricularmente, en el pregrado y el postgrado para perfeccionar la enseñanza del Derecho y específicamente el Derecho sobre Bienes, en materia de equidad de género.

\section{Referencias}

Ahr, I. (2007). Género y Educación. Cuaderno temático. Perú: Ed. EBRA E.I.R.L.

Álvarez de Zayas, C. M. (1999). Didáctica: la escuela en la vida. 3ra edición corregida y aumentada. La Habana: Editorial Pueblo y Educación.

Camargo, J. (1999). Género e Investigación Social. Curso de Formación en Género. Módulo 2. Instituto de la Mujer de la Universidad de Panamá/ UNICEF. Editora Sibauste, Primera edición. Acceso en: sept. 2016. Disponible en: http://www.legalinfopanama.com/articulos/articulos_21c.htm

Caram, T. (1996). La Mujer cubana y la participación social: educación y ciencia. 1996. Tesis de Maestría, Programa FLACSO Cuba, Universidad de La Habana.

Caram, T. (2016). Oportunidades y posibilidades para el empoderamiento. Revista Estudios del Desarrollo Social: Cuba y América Latina, 4 (4), Número Extraordinario, pp. 176-189. Disponible en: 
1503 Silva, Pérez \& Delgado - Analizando la Masculinidad

http://www.revflacso.uh.cu/index.php/EDS/article/view/147. Acceso el: 25 mar. 2017.

Cuba. Constitución de la República de 24 de febrero de 1976 (actualizada con la Reforma de 1992). Disponible en: http://www.acnur.org/fileadmin/scripts/doc.php?file=fileadmin/Docu mentos/BDL/2001/0511

Cuba. Ley No. 65. Ley General de la Vivienda de 23 de diciembre de 1988. En Colección de textos legales. La Habana: Ediciones ONBC.

Diccionario de la Lengua Española, Edición del Tricentenario. (2014).Palabra proceso. Disponible en: http://dle.rae.es/?id=UFbxsxz. Acceso el: 7 jul. 2017.

Horruitiner, P. (2007). La Universidad cubana: el modelo de formación. Capítulo II. El proceso de formación. Sus características. Pedagogía Universitaria, Vol. XII, No. 4: p. 13.

Izquierdo, M J. (1998). El malestar en la desigualdad. España: Ed. Cátedra. De la Universidad de Valencia.

Lamas, M. (2014). La perspectiva de género.Disponible en: http://www.latarea.com.mx/articu8/lamas8.htm. Acceso en: 9 mar. 2017.

Lagarde, M. (1994). La regulación social de género: el género filtro de poder. México. Consejo Nacional de Población.

Miranda, B.\& Peña, V. (2001). Relaciones de Genero con Equidad: Guía conceptual y metodológica. Editorial IICA-Holanda: p. 77.. Disponible en: http://repiica.iica.int/docs/B1178e/B1178e.pdf. Acceso en: 9 mar. 2017.

Páez, L. (2014). Modelo de formación de estudiantes de derecho para la litigación. 2014. Una estrategia para su implementación en la Universidad de Pinar del Río. Tesis de Maestría no publicada, Universidad "Hermanos Saíz Montes de Oca", Pinar del Río, Cuba.

Pautassi, L. (2012). La igualdad en espera: el enfoque de género. Lecciones y Ensayos, Número 89.

Proveyer, C. (2005). Selección de lecturas de Sociología y Política Social de Género. La Habana: Editorial Félix Varela.

Stoller, R. (1994).Sex and Gender: On the Development of Masculinity and Femininity, Science House, New York City. Disponible en: 
http://us.karnacbooks.com/product/sex-and-gender-the-developmentof-masculinity-and-femininity/22/

Scout, J. (1990). El género: una categoría útil para el análisis histórico. Disponible en: http://www.cholonautas.edu.pe/modulo/upload/scott.pdf

Silva, J. L., Páez, L. \& Simón, A. (2016). La relación entre Derecho Internacional Público -Seguridad Nacional para el estudiante de Derecho en Cuba. Universidad y Sociedad [seriada en línea], 8 (4), pp. 149-155.

Recuperado

de

http://scielo.sld.cu/scielo.php?script=sci arttext\&pid=S221836202016000400020

Silva, J. L. \& Pérez, A. (2017). Enfoque de género en la evolución del ordenamiento jurídico cubano y su manifestación en el Derecho Penal actual. Revista Estudios del Desarrollo Social: Cuba y América Latina, Vol. 5, No. 2, Mayo-Agosto, pp. 1-11. Disponible en: www.revflacso.uh.cu

Silva, J.L., Pérez, A. \& Páez, L.D. (2017). La formación del profesional desde el enfoque de género en el Derecho Penal cubano. Revista de Educación y Derecho. No. 16, septiembre de 2017. Disponible en: http://revistes.ub.edu/index.php/RED/index

Staff, M. (1999). Género y Derecho. Curso de Formación en Género. Módulo 3. Instituto de la Mujer de la Universidad de Panamá. Primera edición. Legalinfo, Panamá: Editora Sibauste,. Disponible en: http://www.legalinfopanama.com/articulos/articulos 21f.htm

Valdebenito, E. (2002). Género y Desarrollo (algunas reflexiones y un glosario práctico para facilitar el trabajo a nivel local). Programa DelNet - Gender Equality - Centro Internacional de Formación de la OIT: pp. 37 y 44;. Disponible en: http://www.itcilo.it/delnet

Vasallo, N. (2004). El género: un análisis de la "naturalización" de las desigualdades. EnColectivo de autores. Heterogeneidad social en la Cuba actual Centros de Estudio y Bienestar Humano. Universidad de La Habana. Cuba. 
1505 Silva, Pérez \& Delgado - Analizando la Masculinidad

Jorge Luis Silva-González Professor at Department of Public International Law, "Hermanos Saíz Montes de Oca" de Pinar del Río University, Cuba.

Alie Pérez-Véliz Professor at Department of Law, "Hermanos Saíz Montes de Oca" de Pinar del Río University, Cuba.

Yudmila Delgado-Rodríguez Professor at Department of Law, "Hermanos Saíz Montes de Oca" de Pinar del Río University, Cuba.

E-mail address: silva@upr.edu.cu 